Service social

\title{
Modèles d'éducation, virilité ostentatoire et déficit d'expression de l'intime dans la construction sociale de la masculinité en Martinique
}

\section{Roger Cantacuzène}

Volume 59, numéro 1, 2013

URI : https://id.erudit.org/iderudit/1017484ar

DOI : https://doi.org/10.7202/1017484ar

Aller au sommaire du numéro

Éditeur(s)

École de service social de l’Université Laval

ISSN

1708-1734 (numérique)

Découvrir la revue

Citer cet article

Cantacuzène, R. (2013). Modèles d'éducation, virilité ostentatoire et déficit d'expression de l'intime dans la construction sociale de la masculinité en Martinique. Service social, 59(1), 129-144. https://doi.org/10.7202/1017484ar
Résumé de l'article

L'analyse des entretiens d'une enquête exploratoire sur la socialisation masculine conduit à émettre l'hypothèse que la socialisation des hommes martiniquais est marquée par la quasi-absence d'apprentissage de l'expression de l'intime et la sur-expression codifiée de la virilité ostentatoire. Ainsi, le jeune mâle ne bénéficierait pas de la transmission d'un mode de communication lié à l'intime, les cadres traditionnels de l'apprentissage relationnel constituant des vecteurs de répression ou de refoulement de l'intime.

Ce non-apprentissage ou ce refoulement de l'expression de l'intime ont un coût élevé en termes de mal-être personnel et de conflictualité interpersonnelle. 


\section{Modèles d'éducation, virilité ostentatoire et déficit d'expression de l'intime dans la construction sociale de la masculinité en Martinique}

Roger Cantacuzène

\section{RÉSUMÉ}

L'analyse des entretiens d'une enquête exploratoire sur la socialisation masculine conduit à émettre l'hypothèse que la socialisation des hommes martiniquais est marquée par la quasiabsence d'apprentissage de l'expression de l'intime et la sur-expression codifiée de la virilité ostentatoire. Ainsi, le jeune mâle ne bénéficierait pas de la transmission d'un mode de communication lié à l'intime, les cadres traditionnels de l'apprentissage relationnel constituant des vecteurs de répression ou de refoulement de l'intime.

Ce non-apprentissage ou ce refoulement de l'expression de l'intime ont un coût élevé en termes de mal-être personnel et de conflictualité interpersonnelle.

Mots-clés : Socialisation masculine; éducation; communication; virilité ostentatoire; réputation.

\section{SUMMARY}

The analysis of interviews from an exploratory investigation on male socialization leads to the hypothesis that the socialization of Martinican men is marked by the near-absence of learning to express or reveal the intimate self and by the codified over-expression of an ostentatious virility. Thus, the young male does not benefit from the transmission of communication modes related to the revealing of his intimate self, with the traditional frameworks of relational learning constituting the vectors of the suppression or holding back of such intimateness. However this non-learning or repression of expressing the intimate self have a high cost in terms of personal discomfort and interpersonal conflict.

Keywords: Male socialization; education; communication; ostentatious virility; reputation.

Cette foule criarde si étonnamment passée à côté de son cri $[\ldots]$ cette foule si étrangement bavarde et muette.

Aimé Césaire, Cahier d'un retour au pays natal 
Alors que, dans les sociétés antillaises, la « parole masculine » semble omniprésente dans les diverses sphères de l'espace public et privé, l'analyse des entretiens d'une enquête exploratoire sur la socialisation masculine à la Martinique ${ }^{1}$ nous conduit à émettre l'hypothèse que la communication dont l'individu mâle est acteur incorpore fortement les codes d'une masculinité impliquant le caractère ostentatoire d'une virilité hétéronormée. Le revers de cette «surparole virile » semble cependant reposer sur un «tabou » de l'intime, dont l'expression ne fait l'objet d'aucun apprentissage spécifique tout au long de la socialisation. Par « expression de l'intime », nous entendons ici celle des sentiments profonds, du ressenti profond, l'expression de « ce que je ressens vraiment », indépendamment de la pression de « ce que l'on attend de moi ». Une véritable «expression de l'intime » implique à la fois une démarche introspective de compréhension de soi-même, et une capacité à dire ce ressenti dans les divers cadres relationnels où l'on est investi (couple, famille, amis). Elle suppose également que ces cadres relationnels soient suffisamment empreints de confiance entre les interlocuteurs. Dire l'intime, c'est se dévoiler, se révéler dans ce qu'il y a de plus sensible en soi, c'est manifester non seulement ses désirs, mais aussi laisser transparaître ses faiblesses, ses doutes, ses angoisses. Or, il apparaît que, pris dans ces différentes dimensions le cadre de socialisation des «mâles » martiniquais ne laisse pas de place réelle à un investissement dans la « communication de l'intime ».

Ce déficit d'expression de l'intime, s'il touche particulièrement les hommes, n'est probablement pas absent des formes relationnelles impliquant les femmes. Et l'un de ses ressorts anthropologiques cachés est sans doute la transmission, par delà les générations, d'habitus qui se sont forgés dans la rudesse de l'univers plantationnaire colonial.

Variante micro-insulaire du système de plantation, « la société d'habitation ${ }^{2}$ » créée dans la période esclavagiste a fortement structuré les rapports sociaux au sein des sociétés antillaises (Petitjean-Roget, 1980). Le maintien durable du système esclavagiste n'a cependant été rendu possible que par l'organisation d'un processus de « créolisation » de la main-d'œuvre d'origine africaine, processus destiné à lui faire intérioriser les valeurs d'un ordre reposant sur les rapports maître-esclave, le travail contraint, et plus généralement «les formes culturelles encouragées et sélectionnées par les maîtres » (Gauthier, 2007, p 13-14). La société d'habitation a ainsi contribué à produire des normes sociales qui informent encore les sociétés

1. La recherche «Genre et violences interpersonnelles à la Martinique », menée sous la direction de Nadine Lefaucheur (CNRS, CRPLC/UAG) et d'Elizabeth Brown (Institut de Démographie, Université Paris I - PanthéonSorbonne), dans le cadre d'un programme ANR, a comporté trois volets : une étude statistique, de type ENVEF (enquête nationale sur les violences faites aux femmes) mais étendue aux violences subies par les hommes, portant sur un échantillon de 1000 femmes et 1000 hommes âgés en 2008 de 18 à 59 ans, et deux enquêtes par entretiens approfondis, l'une auprès de femmes victimes de violence (20 femmes), l'autre, à caractère exploratoire, portant sur la socialisation masculine et la violence à la Martinique (19 hommes). Les entretiens de cette dernière ont été recueillis par Roger Cantacuzène, Joëlle Kabile, Paola Lavra, Clara Palmiste et Mylenn Zébina-Zobda en 2008-2009. Les résultats de l'enquête statistique et certaines conclusions de l'enquête qualitative relative aux femmes victimes de violence ont déjà été publiées (Lefaucheur, dir., 2011). Les analyses relatives à l'enquête sur la socialisation masculine ont fait l'objet de plusieurs communications en voie de publication et d'une contribution à l'ouvrage Boys don't cry! (Lefaucheur et Mulot, 2012).

2. Dans l'aire tropicale des Amériques, les plantations constituaient des entités économiques reposant sur le travail des esclaves et visant la production intensive de certaines cultures (notamment le sucre). Portant sur de grandes exploitations, elles admettaient l'absentéisme des maîtres. Dans les petites Antilles, ces entités, plus petites, où le maître (appelé « l'habitant ») était généralement présent, portaient le nom d'habitation. 
dites « créoles » antillaises, qu'il s'agisse des représentations hiérarchisant les nuances de couleur de peau (Bonniol, 1992), ou encore des rapports de sexe valorisant le rôle de l'homme comme géniteur et sacralisant la femme, à travers la matrifocalité, dans un rôle de mère/père poto-mitan ${ }^{3}$, la vouant à se sacrifier aux besoins de sa famille (Gracchus, 1980 ; André, 1987 ; Mulot, 2000). Par-delà les mutations socio-économiques qu'ont connues les Antilles, certaines marques des processus initiaux de «créolisation » imprègnent encore les formes relationnelles et se cristallisent dans des styles d'éducation ou de communication perçus comme « allant de soi ».

L'analyse proposée ici repose principalement sur le dépouillement du corpus des 19 entretiens approfondis de l'enquête qualitative exploratoire «Socialisation masculine et violence ${ }^{4} »$, tout en prenant en compte certaines informations complémentaires fournies par les deux autres volets de la recherche «Genre et violences interpersonnelles à la Martinique », à savoir l'enquête statistique et l'enquête qualitative relative aux femmes victimes de violence (Lefaucheur, 2011).

Les entrevues de l'enquête « socialisation masculine et violence » ont été obtenues auprès d'hommes martiniquais dont l'essentiel de la socialisation, tant primaire que secondaire (Berger et Luckman, 1966), s'est déroulée en Martinique : ils y sont nés, y ont grandi et y avaient vécu, au moment de l'enquête, la majeure partie de leur existence (en dépit, pour six d'entre eux, de périodes de migration plus ou moins longues liées aux études ou à la recherche d'un emploi, comme cela est fréquent pour les jeunes Antillais). La prise de contact avec les enquêtés s'est opérée de manière aléatoire, les enquêteurs optant quelquefois pour des inconnus abordés pour les besoins de l'enquête (3 cas), mais le plus souvent pour des personnes appartenant à leur entourage personnel ou professionnel, même si le degré de connaissance interpersonnelle préalable était limité (ami d'ami, mécanicien, personne rencontrée par ailleurs dans un cadre professionnel...). Une coordination régulière assurée au sein de l'équipe a permis de disposer d'un éventail large en termes de représentation d'âge ${ }^{5}$ (de 22 à 68 ans), de statuts matrimoniaux (célibataires, personnes en situation de couple mariés ou non, avec ou sans cohabitation, divorcés) ou parentaux (pères de famille ou non), de niveau d'études, de religion (catholicisme et protestantisme essentiellement, mais évocation du bouddhisme et du rastafarisme dans deux cas), d'orientation sexuelle (2 enquêtés homosexuels et un bisexuel). La forte proportion des personnes de moins de 40 ans interrogées (14 sur 19, dont 6 de moins 30 ans) laisse présager du caractère encore récent et extrêmement prégnant des formes de socialisation relevées. Conduites de manière semi-directive, en face-à-face, sur le fondement d'un guide d'entretien prédéfini, les entrevues ont permis d'obtenir des récits biographiques renseignant plusieurs champs: socialisation intrafamiliale, scolarité, déménagements/migrations, «premières fois » (rapports sexuels, emploi, tabac, alcool, drogues, etc.), service militaire, mises en couple, mariages, séparations, paternités,

3. Le « poto-mitan » est le pilier central de la « case », maison rudimentaire construite en bois et terre jusqu'à la fin des années 1950. L'expression «femme poto-mitan » est fortement présente dans les sociétés antillaises, imageant la représentation du rôle de la femme, considéré comme essentiel, dans la famille.

4. L'enquête s'est déroulée sous forme d'entretiens semi-directifs, chacun des enquêteurs disposant d'un guide d'entretien préalablement préparé par l'équipe de recherche.

5. Pour des raisons à la fois juridiques et de mise en corrélation avec l'enquête statistique, seuls des individus majeurs ont été interrogés. 
changements professionnels, migrations, conversions religieuses, condamnations, faits de violence à titre de témoin, de victime ou d'auteur, etc.

Cet article se propose d'interroger, à travers les divers cadres relationnels abordés dans l'enquête (famille, école, groupes de pairs), les modèles éducatifs qui président à la socialisation masculine et qui concourent à produire une surexpression de la virilité codifiée sous une forme ostentatoire, tandis que l'expression de l'intime, prise entre désir et malaise, demeure passablement sous-développée.

Cette faible propension à exprimer l'intime est à rattacher à des formes d'éducation et de communication interpersonnelle qui, imprégnant l'ensemble de la société, se perpétuent dans une sorte d'impensé social, ou sont implicitement perçues comme utiles car «traditionnelles » (1).

Les vecteurs de construction de la masculinité viennent cependant renforcer le refoulement de l'intime, tandis qu'ils accentuent l'orientation de la communication masculine vers le caractère ostentatoire de la virilité (2). Un tel « effacement de l'intime » entraîne des coûts importants, en termes de mal-être individuel et de difficultés interpersonnelles (3).

\section{STYLE D'ÉDUCATION ET REFOULEMENT DE L'INTIME}

\section{Un style d'éducation « traditionnel » à dominante autoritaire ?}

Dans la relation adulte/enfant, le style éducatif de « l'autorité imposée » apparaît comme le plus prégnant. La relation parent/enfant est le cadre privilégié de ce modèle, qui est souvent perçu comme un style d'éducation « traditionnel » et transgénérationnel : il ressort en effet de tous les récits biographiques des enquêtés, à l'exception d'un seul d'entre eux, et cela quels que soient leur âge et leur statut socioéconomique. Il a également gouverné la scolarité (surtout au primaire) de nombre d'entre eux, notamment les plus âgés.

Ce modèle postule l'obéissance attendue de l'enfant comme allant de soi, l'adulte se présentant comme un pôle d'autorité indiscutée et indiscutable. II repose sur quatre éléments principaux, qui se manifestent à des degrés divers dans les récits de vie: l'injonction (concernant un comportement attendu, sans discussion); les punitions et corrections corporelles (à titre de sanction); le faible recours à l'encouragement, à la récompense et à la valorisation ; la place réduite accordée à l'écoute, au dialogue.

\section{Injonctions et punitions corporelles : deux piliers de l'autorité imposée}

Injonctions et sanctions corporelles sont les deux piliers principaux de l'affirmation de l'autorité de l'adulte. La parole injonctive de l'adulte porte, quant au contenu, sur un comportement ou une action attendue de l'enfant, sans discussion possible. Mais c'est aussi la forme, les modalités non verbales de cette parole injonctive qui semblent devoir lui conférer la force de l'autorité : le ton de la voix et son volume sonore montent d'autant plus en amplitude que l'on veut «faire entendre » l'autorité. Dans une certaine mesure, l'exercice d'une autorité « forte » équivaut à celle d'une autorité « bruyante ». 
Par ailleurs, imposer l'autorité de l'adulte, c'est également avoir recours à des sanctions, qui seront fréquemment des corrections corporelles. Le rôle de l'autorité dispensatrice de ces punitions semble socialement dévolu au père dans les cas où celui-ci est présent au foyer. Cependant cette «fonction » est très facilement reprise et exercée par la mère. Dans le contexte de la matrifocalité antillaise qu'a pu décrire Stéphanie Mulot (2000), la mère fait largement figure d'autorité, notamment pour tout ce qui a trait à l'éducation des enfants. Aussi le recours aux corrections corporelles administrées par la mère se présente-t-il comme une pratique courante.

C'est surtout l'omniprésence du recours à l'objet (liane, ceinture, bâton, corde, câble électrique, etc.) qui, dans les récits biographiques, interpelle. Objet visible, objet « marquant » du corps et de la psyché, symbole de l'autorité, l'instrument du châtiment ne se présente-t-il pas aussi comme le succédané de la « liane » du commandeur d'habitation ? Dans ses travaux sur la société d'habitation, Jacques Petitjean-Roget évoque le barème relevé par le missionnaire et chroniqueur Du Tertre concernant les châtiments applicables dans la société coloniale esclavagiste, lequel s'inspire de l'arsenal de répression en vigueur en France au XVII siècle (Petitjean-Roget, 1980). Si les fautes les plus graves donnent lieu à des supplices exécutés par un bourreau désigné parmi les esclaves, les châtiments les plus ordinaires s'intègrent à la vie quotidienne des esclaves et sont exercés sur eux avec rigueur. Ainsi « la paresse » est-elle sanctionnée par le commandeur avec « la lianne qu'il porte ordinairement à la main; elle fait autant ou plus mal qu'un nerf de bœuf ». De même, pour la désobéissance, «l'on n'épargne point les coups de lianne au nègre qui refuse de faire ce qui luy est commandé » (Du Tertre, cité par Petitjean-Roger, 1980, p. 1136).

Et, tandis que Petitjean-Roget rappelle que tout cet arsenal est destiné au « bon dressage » des individus réduits en esclavage, l'on pourra se demander jusqu'à quel point l'image du « sauvage à dresser », si vivace dans l'univers colonial esclavagiste, a pu imprégner le style d'éducation, lequel s'est retransmis de génération en génération, au point d'apparaître comme le modèle « traditionnel » :

Mon père nous voyait comme des enfants indisciplinés, des petits sauvages qu'il fallait discipliner. II avait une grosse ceinture en cuir, il a enlevé la boucle et c'est avec cela qu'il nous frappait régulièrement. II nous voyait comme des enfants mal élevés, qui criaient, qu'il fallait dompter. (Christian, 27 ans, éducateur spécialisé).

\section{Manque de valorisation et place réduite accordée à l'écoute: deux vecteurs d'une « communication » insatisfaisante}

Dans la mesure où l'autorité de la parole de l'adulte est considérée comme « allant de soi », l'un des corollaires du style d'éducation « traditionnel » est la minoration de la place accordée à ce que ressent le destinataire du message. Cette négation du ressenti de l'enfant fait qu'il est accordé, dans l'éducation, une faible place aux encouragements, à la valorisation des efforts. De plus, les soucis personnels des parents, leur manque de temps ou la fatigue, mais aussi l'absence d'un modèle de relations entre adultes et enfants autre que celui de l'autorité imposée, limitent leur disponibilité pour l'écoute, le dialogue, l'échange. Si ces dernières modalités ne sont guère inscrites dans le répertoire du style d'éducation considéré comme 
traditionnel, ce n'est point qu'elles en soient, d'emblée, totalement exclues par les parents, mais plutôt qu'elles n'ont pas fait l'objet d'un apprentissage.

\section{Des comportements de résistance au conflit de générations ?}

Les formes que revêt le style d'éducation traditionnel peuvent sembler relativement bien établies et acceptées par les individus, au fil des années et de l'intégration par eux des normes de leur socialisation. Lors de l'enquête statistique «Genre et violence à la Martinique » (Lefaucheur, 2011 ; Lefaucheur et Mulot, 2012), trois hommes et deux femmes sur dix ont ainsi déclaré être «d'accord » avec la proposition « pour bien élever un enfant, il faut le corriger physiquement». De même, si, à la question «au cours de votre enfance ou de votre adolescence, avez-vous été fréquemment puni ou battu injustement ? », une personne sur cinq a répondu positivement, nombreuses ont été, parmi celles qui ont répondu négativement, celles qui ont précisé : «J'ai souvent été frappé, mais ce n'était pas injuste puisque c'était pour mon bien. " Les débats radiophoniques ou télévisuels qui ont suivi la publication de l'enquête statistique ont également souligné la forte persistance dans l'opinion martiniquaise du modèle de l'autorité imposée par le recours aux châtiments corporels et la crainte largement répandue que sa mise en cause par de nouvelles normes éducatives ne soit responsable d'une « envolée » de la délinquance aux Antilles.

Cependant, l'enquête exploratoire sur la socialisation masculine a montré que les modes de communication rattachés au style d'éducation autoritaire entraînaient aussi des tensions et des comportements de résistance, qui pouvaient éventuellement se traduire par des actes délinquants, notamment lorsqu'ils induisaient un sentiment d'injustice ne pouvant s'exprimer frontalement face à la parole adulte dominante ${ }^{6}$.

Ainsi, les mots de l'adulte ou son comportement, tels qu'ils sont perçus par l'enfant ou le jeune, apparaissent quelquefois au moins aussi blessants pour ce dernier que les punitions physiques. Dans certains cas, le silence observé par l'enfant peut s'accompagner de réactions où le ressenti s'exprime de manière détournée, sous une forme non verbalisée, et parfois violemment :

Une fois, j'avais mis le feu à la maison (Marc, 25 ans, étudiant).

La difficulté à établir une communication interpersonnelle prenant en compte le ressenti de l'enfant (puis, plus tard, du jeune) tend donc à produire des mésententes adulte-enfant, adultejeune. Perçues parfois comme la résultante d'un « conflit de générations », certaines attitudes de rébellion contre toute forme d'autorité sont sans doute elles-mêmes une forme de réaction vis-à-vis d'un style de communication intergénérationnelle insatisfaisant.

6. Plusieurs enquêtés se montraient d'ailleurs critiques à l'égard du style d'éducation auquel ils avaient été soumis et envisageaient ou s'efforçaient de ne pas le reproduire à l'identique avec leurs enfants : « On ne nous tapait pas pour rien. C'était pour notre bien, après. Mais quand même, je dis que la violence, un enfant ne devrait pas connaître ça. Quand tu reçois les coups, tu ne comprends pas. Il y a d'autres méthodes, je pense. » (François, 23 ans, employé). 


\section{LE DÉFICIT D'EXPRESSION DE L'INTIME DANS LA CONSTRUCTION DE LA MASCULINITÉ}

Soumis à la forte contrainte socialement imposée aux mâles antillais de se construire une « réputation virile », le fonctionnement des groupes d'hommes est structuré par l'expression ostentatoire de la virilité, qui se présente donc comme un mode de communication transmis par les pairs, autant sinon plus que par les aînés. La survalorisation d'une communication tournée vers l'ostentation de la virilité se fait cependant au détriment d'autres champs d'expression qu'elle laisse dans l'ombre, n'accordant guère de place à l'expression d'une parole intime.

\section{Le groupe des pairs : espace d'expression de la virilité ostentatoire}

Les entretiens de l'enquête sur la socialisation masculine à la Martinique viennent confirmer les observations faites dans la Caraïbe sur la construction des normes de genre. L'injonction de virilité qui s'impose à l'individu mâle (Mulot, 2009 ; Lefaucheur et Mulot, 2012) est le noyau fondamental du critère de « réputation » repéré par des études de genre caribéennes. La « réputation » est en effet, avec la « respectabilité », l'un des volets d'un double standard normatif mis en évidence par Peter Wilson $(1969,1973)$ pour la Caraïbe anglophone, puis repris dans des études liées à la Caraïbe francophone (André, 1987 ; Giraud, 1999 ; Mulot, 2000, 2009 ; Bougerol, 2002 ; Kabile, 2011 ; Lefaucheur et Mulot, 2012).

Selon les travaux de Wilson, effectués sur l'île anglophone de Providencia, la « respectabilité » caractériserait essentiellement les normes comportementales s'imposant dès l'enfance aux femmes, tandis que la « réputation » présiderait à la socialisation masculine. La respectabilité s'appuierait, selon lui, sur des valeurs héritées de l'organisation coloniale et destinées à la reproduire, au premier rang desquelles se retrouveraient la moralité, la religion, l'éducation. La réputation, serait, en revanche, un ensemble de valeurs indigènes présentes essentiellement chez les hommes dans les communautés de pairs et produites en adaptation à un univers caribéen esclavagiste puis post-esclavagiste. Elle impliquerait, entre autres, la défense de l'honneur, de la sagesse, de la capacité à se valoriser par des talents de parleur ou d'artiste. Mais elle repose aussi sur l'aptitude à démontrer sa force et sa virilité, notamment, pour cette dernière, à travers la multiplication de conquêtes féminines, et par la narration des exploits sexuels.

Plusieurs marqueurs d'un « langage » de la virilité ostentatoire se rattachant à la réputation peuvent être repérés à travers l'analyse du discours des enquêtés. Chez les jeunes hommes, et notamment au sein du groupe des pairs, les manifestations de force, les expressions de rivalité font partie des épreuves de masculinité habituelles. Ainsi, savoir se battre, «avoir son respect », permet d'être reconnu et valorisé au sein du groupe. De plus, le recours aux manifestations de force peut être considéré comme un atout supplémentaire de séduction. Un autre marqueur porte sur la sexualité et sur la manifestation au regard du groupe de sa capacité à séduire, à avoir plusieurs conquêtes féminines (capacité souvent matérialisée par des progénitures multiples). Si la mise en scène (narration des exploits) s'opère essentiellement à destination du groupe des pairs dont le regard est indispensable, des incitations fortes proviennent également des aînés masculins. 
La virilité ostentatoire implique cependant de masquer l'intime en ce qu'il révèle comme faiblesse. Des émotions de peur, le «trac», la timidité peuvent alors être surmontés par certaines pratiques considérées comme viriles, telles le recours à l'alcool. Pour manifester de la force, le jeune mâle doit gommer au regard de l'autre, du groupe, les faiblesses, les angoisses, les doutes, mais aussi les sentiments trop délicats. La virilité ostentatoire est une forme de communication. Mais celle-ci est tournée vers la séduction («collectionner les filles »), le paraître, «l'épate ». Apprenant à séduire et à conquérir, le garçon développe plus ou moins bien des habiletés en ce sens. En revanche, cet apprentissage n'implique pas, voire interdit, de se dévoiler en profondeur, d'être « vrai ».

L'apprentissage des codes de la virilité ostentatoire ne sollicite, par ailleurs, nullement la capacité à communiquer dans une situation conflictuelle. Ce modèle de communication butte donc sur ses propres limites : s'il facilite sans doute l'établissement d'une relation de couple, il permet difficilement le maintien harmonieux de cette relation.

\section{Absence de transmission par les aînés masculins d'un modèle de communication de l'intime}

L'analyse des entretiens fait aussi ressortir l'absence de transmission par les aînés masculins, et en particulier par le père, d'un modèle de communication où s'exprimerait le ressenti intime des individus.

Quelquefois, le modèle de communication que transmet le père se fonde uniquement sur la virilité ostentatoire. C'est le cas du père de Manuel, soucieux d'exhiber devant lui ses conquêtes féminines, mais qui ne semble pas vraiment s'intéresser à ce que vit son fils :

Il venait : «Alors, ça va ? - Ça va. - Et l'école, ça va ? » C'était pas quelqu'un qui donnait des conseils et tout ça (Manuel, 34 ans, travailleur social).

Plus généralement, la forme de communication du père, héritée du modèle de socialisation masculine, tend à limiter la manifestation de marques d'affection vis-à-vis du fils. Cette retenue affective est opérante même lorsqu'il s'agit d'un «père présent », ou d'un père considéré comme remplissant son rôle paternel, parce qu'il subvient aux besoins matériels de sa famille ou de ses enfants. En effet, qu'il soit « le cuistot de la baraque » (Jean-Jacques, 30 ans, agent d'exploitation), un père « jouant son rôle » (François), un père « gentil, généreux » (Marc) ou un « bosseur » (Jean-Pierre, 44 ans, mécanicien; Bernard, 35 ans, enseignant), c'est sur le plan de l'interaction affective et relationnelle que semble se jouer l'une des dimensions de «l'absence ». Cette distance affective met alors le père, même présent au foyer, dans une situation de présence-absence, puisqu'il est à la fois présent (par les tâches dont il s'occupe) et absent (par insuffisance de communication) :

C'est pas qu'on le voyait pas du tout, mais il était plus occupé à vaquer à ses affaires, je sais pas (Jean-Jacques).

C'était un père absent, ce n'était pas un père qui passait du temps avec ses enfants, il ne nous connaissait pas (Christian). 
Le sentiment « d'absence » peut, certes, être lié à une insuffisance de moments partagés, même lorsque le père vit dans le foyer. Mais c'est aussi la faible propension masculine à verbaliser et à échanger sur des sujets intimes qui induit une impression de distance affective. Faute d'un apprentissage de formes de communication facilitant l'expression libre des sentiments, les échanges restent centrés sur des savoirs pratiques plutôt que sur le ressenti ou les préoccupations des individus. La distance affective que crée ce modèle relationnel fait alors qu'il y a peu de chances que le père constitue pour son fils un confident.

L'une des principales limites de ce modèle est donc qu'il n'offre guère d'espace de dialogue sur des sujets s'écartant des savoirs pratiques (travail, jobs, mécanique, etc.) ou autres que ceux liés à la virilité ostentatoire, notamment ceux pouvant entraîner un dévoilement de l'intime.

\section{LES COÛTS DE “ L'EFFACEMENT DE L'INTIME " : MAL-ÊTRE INDIVIDUEL ET DIFFICULTÉS INTERPERSONNELLES}

\section{Le mal-être individuel ou la solitude en groupe}

Structurées autour d'un véritable «tabou de l'intime», les formes qu'emprunte la communication masculine ne sont pas sans lien avec l'existence d'un mal-être qui ne se dit pas, ne se nomme pas, mais qui constitue une souffrance mise en évidence dans des travaux ethnoépidémiologiques (Massé, 2008). En effet, la diversité des espaces de mise en scène de la parole masculine de même que le caractère ostentatoire de cette dernière masquent la fragilité de la construction d'une image sociale et d'une identité de genre ne laissant pas de place à l'expression de la sensibilité. Sommé d'être fort, d'être viril, l'homme le sera sous le regard du groupe social, en cuirassant son attitude, en gommant extérieurement les aspérités de ses points sensibles, en taisant autant que faire se peut, le cas échéant, ses souffrances.

\section{Occulter toute faiblesse : le déni de souffrance}

Les éléments fondateurs de ce modèle de construction de la masculinité se mettent en place dès l'enfance, et l'enfant mâle devra très tôt apprendre à s'y conformer afin de s'adapter aux codes sociaux dominants. Les enquêtés ayant été témoins de violence au sein de leur milieu familial ont ainsi dû se créer des stratégies comportementales afin de masquer leurs angoisses.

Rudy se souvient encore d'une période douloureuse, alors qu'il était âgé de 10-11 ans, où il a été témoin des menaces du compagnon de sa mère envers celle-ci. L'enfant qu'il était vivait cette situation avec une angoisse, qu'il tentait d'apaiser en mettant en place une stratégie d'occultation. La posture stoïque adoptée par cet enquêté est cependant conforme aux codes de la virilité visant à gommer l'expression de la faiblesse, attitude débouchant in fine sur un déni de la souffrance :

Une fois que j'étais sorti du milieu, du foyer, j'oubliais, je mettais un écran total, j'avais complètement oublié ce qui se passait. C'est en rentrant, l'angoisse revenait. Mais tu mets une barrière, tu ne penses pas à ça (Rudy, 39 ans, agent administratif). 
La dissonance entre les déclarations et l'attitude non verbale se révèle parfois pourtant troublante. Dans son récit, Jean-Jacques, dont les parents avaient des relations conflictuelles comportant beaucoup de violence verbale, indique :

Les parents s'engueulaient tout le temps, avec toutes les insultes qu'on connaît. Y avait énormément de violence verbale à la maison, mais il n'y a jamais eu de violence physique. C'est plutôt les filles qui écoutaient ça, moi, je m'en foutais. Rien ne m'atteignait. Je prenais ma moto et j'allais voir mes copains.

S'il se forge une «carapace » pour ne pas être atteint par cette atmosphère conflictuelle, son attitude de fuite trahit sans doute le fait qu'il était en réalité très affecté par cette situation. Mais l'espace qu'il rejoint pour éviter de ressentir cet « intime » douloureux est le groupe des pairs, espace peu adapté à son expression et donc, a fortiori, au traitement des souffrances éprouvées.

\section{L'injonction d'invisibilité de l'homosexualité}

D'autres aspects de l'intime peuvent également être soigneusement dissimulés, précisément parce qu'ils dérogent de manière trop «remarquable » aux prescriptions normatives de construction de la masculinité. C'est particulièrement le cas de l'expression du désir homosexuel. Montrant à quel point, dans un contexte social, familial et religieux stigmatisant l'homosexualité, la découverte de son attirance pour des individus du même sexe pouvait être perçue, de manière douloureuse, comme « une anomalie, une discordance entre, d'une part l'obligation de se conformer à la norme hétérosexuelle [...] et d'autre part son désir », le constat fait dans une étude ethnographique auprès d'enquêtés guadeloupéens (Pourette, 2002, p. 55), apparaît tout aussi valable pour la Martinique. L'homosexualité masculine y est perçue comme une remise en cause des critères d'élaboration de la réputation virile (Mulot, 2009), plaçant ainsi les personnes concernées dans les marges sinon hors de la masculinité (Zobda-Zebina, 2011).

L'interdit social pesant très fortement aux Antilles sur l'homosexualité se présente comme une injonction très tôt intériorisée, et imprègne tellement le corps social qu'il peut paraître difficile de distinguer une source injonctive particulière. Cet interdit social, de par son caractère à la fois diffus et fortement chargé émotionnellement - dans le sens d'une stigmatisation radicale - , rend difficile tout débat serein sur l'homosexualité ${ }^{7}$. Dès lors, l'injonction de virilité, qui est au cœur de la socialisation masculine, se traduit pour les hommes homosexuels en une injonction de clandestinité ou d'invisibilité de leur sexualité (Cantacuzène, 2012).

Ainsi, parmi les enquêtés, Marcel, un employé de 44 ans, a toujours dissimulé son orientation sexuelle. Pour sa part, Christian a socialement recours au travestissement de sa vie affective et sexuelle : ainsi, durant l'interview, il adoptera une posture absolument conforme au modèle hétéronormé de la virilité ostentatoire, avant d'avouer, interrogé sur l'homosexualité, et

7. L'ampleur des mobilisations récentes contre la loi étendant le droit au mariage et à l'adoption aux couples homosexuels manifeste bien cette dimension émotionnelle rattachée aux craintes d'effondrement de la masculinité traditionnelle (Cantacuzène, 2012), ainsi que la surdétermination des prises de positions dans l'espace public : trois des quatre députés martiniquais (tous des hommes) et des responsables de la Fédération socialiste de Martinique se sont ainsi déclarés fortement opposés au projet de « mariage pour tous » défendu par le gouvernement socialiste. 
parce qu'il avait été mis en confiance, que lorsqu'il parlait de filles, avec l'enquêtrice comme avec ses collègues, il s'agissait en réalité de garçons.

\section{L'absence d'espace de confidence}

Dans des territoires insulaires se présentant comme des microsociétés, où « tout se sait » et où, au gré des amitiés ou des rivalités interpersonnelles, la parole dite sur soi par l'autre peut se montrer tour à tour bienséante ou malveillante, cette dernière demeure l'un des vecteurs de la construction tant de la réputation que de la respectabilité. Le « qu'en-dira-t-on », le « makrélaj » (commérages) circulant dans les groupes sociaux font en effet office de médias informels mais omniprésents. La parole véhiculée sur soi peut donc représenter un atout tant qu'elle favorise l'ascension vers les idéaux-types de réputation ou de respectabilité, mais aussi un danger lorsqu'on s'éloigne de ces idéaux-types ${ }^{8}$.

En prenant en compte ce contexte, il n'apparaît pas étonnant que les individus soient réticents à exprimer l'intime, comme le suggère l'adage populaire « sé kouto sèl ki sav sa ki an tyè jiromon » (seule la lame du couteau connaît les secrets du cœur d'un potiron). Si la faible propension à exprimer l'intime semble impliquer tant les hommes que les femmes, elle affecte davantage les premiers. Lors des entretiens téléphoniques de l'enquête statistique, un homme sur deux, contre une femme sur trois, a ainsi répondu n'avoir personne à qui se confier en cas de problème personnel.

C'est sans doute un indice que les cadres sociaux de construction de la masculinité se révèlent, en fin de compte, d'une certaine rigidité et n'offrent pas d'espaces pouvant être perçus comme suffisamment bienveillants pour faire tomber les « barrières ». Cette situation explique que l'un des enquêtés, âgé de 34 ans, ait été victime de sévices sexuels durant son enfance et son adolescence, sans pouvoir en parler à quiconque.

\section{Le groupe des pairs, obstacle à l'expression de l'intime ?}

Dans la mesure où un grand nombre de garçons vivent toujours en bande, ils n'y trouvent pas l'espace pour y exprimer leurs émotions profondes, leurs doutes, leurs pensées intimes. Car la parole qui circule au sein du groupe n'est pas celle du ressenti profond, mais uniquement celle qui correspond aux normes codifiées de la virilité ostentatoire. La communication se résume alors à un jugement négatif porté par les pairs, ainsi que l'analyse Bernard qui joue régulièrement au football avec une bande de jeunes:

Ils vivent des expériences communes, mais ils n'ont pas de confidents. Ils ont un problème, ils essaient peut-être d'en parler, mais ils ne s'écoutent pas ou ils connaissent déjà la réponse : «Ah, tu es trop nul! » On ne leur a pas apporté de réponse, hein, on leur a porté un jugement. II n'y a pas d'interrogation, c'est le jugement toujours.

8. Dans une société où le magico-religieux est encore très prégnant, le danger ne concerne pas la seule « réputation », mais aussi l'usage maléfique qui pourrait être fait des confidences sur soi. 
N'ayant pas développé leur capacité à l'introspection et à l'échange sur les sentiments profonds, n'ayant pas de confident avec qui échanger, ces jeunes sont fortement sensibles au jugement des pairs, et désemparés lorsqu'ils doivent prendre seuls certaines décisions. Ainsi, si le groupe de pairs se présente comme l'un des vecteurs structurants de l'identité de genre masculine, il présente aussi l'inconvénient d'être un espace de nivellement des sensibilités, voire parfois un frein à l'expression épanouissante des individualités.

\section{Des difficultés interpersonnelles à l'agressivité à fleur de peau}

Dans la mesure où la parole sur l'intime, contrairement au langage de la virilité ostentatoire, ne dispose pas d'un espace «naturel » d'apprentissage et d'expression dans la socialisation masculine ${ }^{9}$, l'une des conséquences probables est celle d'un style de communication laissant une place importante à l'implicite et à la non-verbalisation des sentiments. Or cela n'est pas sans conséquence sur les relations de couple.

Alors que le champ conjugal pourrait se présenter a priori comme l'un des terrains privilégiés de la communication intime, il est peut-être aussi l'un de ceux qui pâtissent le plus des effets d'une communication inadaptée, notamment dans les périodes conflictuelles. En prolongement de la socialisation de genre duale, reposant sur la «réputation » masculine et sur la « respectabilité » féminine, se cristallisent en effet des attentes de genre différentes quant à la mise en place et au fonctionnement d'une vie de couple.

Pour les hommes martiniquais, préserver les privilèges masculins de liberté sexuelle, de liberté de déplacement et de sorties sans avoir de «comptes à rendre », tout en mettant en place une vie conjugale est un enjeu fondamental. Les attentes féminines, en revanche, semblent centrées sur la capacité à « garder son homme » dans un contexte de compétition sur le marché matrimonial (Mulot, 2000, 2009). Les projections mutuelles relatives au comportement du partenaire sont alors elles-mêmes soumises au jeu de ces attentes contradictoires.

En effet, c'est peut-être sur le terrain du maintien de la « liberté masculine » qu'il est possible de rechercher un facteur explicatif des bénéfices (pour l'homme) supposés être rattachés à la non-exposition de l'intime masculin. Ainsi, l'homme peut être volontiers porté sur le silence et les non-dits en vue de préserver sa liberté sexuelle et de cloisonner les différents segments de sa vie en cas de pluripartenariat ${ }^{10}$ - en application de l'expression créole « sa zyé pa wè, tyè pa fè mal » (ce qui n'a pas été exposé au regard ne crée pas de souffrances). Cependant la propension à la non-verbalisation de l'intime, qui découle des formes de communication rattachées à la socialisation masculine, peut être génératrice d'inquiétude chez sa compagne (Lefaucheur et Ozier-Lafontaine, 2011). Dans un contexte de crainte de la perte du partenaire,

9. II n'est pas exclu que l'obligation de « respectabilité » soit tout autant génératrice d'inhibitions dans la socialisation féminine (Lefaucheur, Ozier-Lafontaine, 2011).

10. Le pluripartenariat masculin se définit comme le fait pour un homme d'avoir plusieurs partenaires avec qui il entretient des relations sexuelles. Les études sociologiques, réalisées dans le cadre des recherches sur la transmission du VIH, révèlent qu'il est nettement plus répandu aux Antilles françaises qu'en France métropolitaine, qu'il y est plus fréquemment simultané et stable (les hommes concernés vivant simultanément plusieurs « relations amoureuses » se prolongeant dans le temps) et plus souvent le fait d'hommes mariés ou vivant en couple (Halfen, 2006). 
les femmes, dans l'espoir de renforcer leur couple, sont alors souvent tentées de vouloir le « contrôler », voire même de le « changer », l'effet obtenu étant cependant une exacerbation des tensions du fait de l'atteinte à une zone particulièrement sensible pour l'homme martiniquais.

L'insuffisant développement d'un mode de communication permettant l'exposition confiante, de part et d'autre, du ressenti intime des individus engendre alors de la difficulté à gérer autrement que sur un mode défensif/agressif les zones potentiellement conflictuelles inhérentes aux relations interpersonnelles, et semble à l'origine de nombre de situations de violence conjugale (Lefaucheur, 2011 ; Lefaucheur et Ozier-Lafontaine, 2011).

\section{Conclusion}

En donnant la parole à des hommes, l'enquête exploratoire portant sur un public-cible masculin a permis de découvrir certains effets insoupçonnés de la socialisation masculine.

La socialisation genrée sur le mode « réputation masculine/respectabilité féminine » intègre de fait, en son sein, un véritable modèle viriliste ${ }^{11}$ qui somme en permanence tout homme d'avoir à démontrer sa virilité. II en découle une forme de communication masculine dominante que nous qualifions de «virilité ostentatoire», dans la mesure où les codes de la communication masculine, intégrés tout au long de la socialisation, sont en permanence soustendus par cette injonction de démonstration. Cependant le talon d'Achille de ces codes de communication, dont la visée est généralement d'obtenir l'approbation ou l'admiration du groupe des pairs, ou encore d'affirmer une capacité de séduction, est la faible propension à exprimer l'intime, le soi profond, notamment en ce qu'il peut révéler de fragile. L'effacement de l'intime dans la communication est d'autant plus important qu'il est favorisé par des modèles d'éducation «traditionnels », encore fortement prégnants, reposant plus sur l'autoritarisme que sur des échanges verbalisés relatifs au ressenti.

Les principaux coûts du modèle de construction de la masculinité et des formes de communication qui y sont associées (surinvestissement dans la virilité ostentatoire, effacement de l'intime) sont, d'une part, des zones de malaise intérieur, lorsque les individus mâles sont confrontés à des situations en dissonance avec les codes virilistes. Ce sont aussi, d'autre part, les difficultés liées à la gestion pacifiée des relations interpersonnelles, notamment lorsque celles-ci comportent une dimension conflictuelle.

$\mathrm{Au}$ regard de la question des violences interpersonnelles, dont celle des violences conjugales, qui ont émergé depuis quelques années en tant que sujet sensible au sein de la société martiniquaise (Lefaucheur, 2011), modifier de manière significative certains traits des styles traditionnels d'éducation et de communication constitue un véritable enjeu de société. Celui-ci souligne la nécessité de politiques publiques portant sur le développement de vecteurs d'éducation relationnelle pouvant influer sur les divers champs de la socialisation.

11. Daniel Welzer-Lang définit le virilisme comme une « exacerbation des attitudes, représentations et pratiques viriles » (Welzer-Lang, 2002). 
A cet égard, des mesures permettant la diffusion, à travers des formations adaptées à divers publics (parents, couples, enseignants...), de formes communicationnelles mieux orientées vers la compréhension de soi et des autres pourraient être envisagées: soutien à l'éducation parentale, formations à la communication non violente, à la gestion des conflits, etc. Elles pourraient passer tant par des créneaux institutionnels existants, publics ou privés (écoles, associations...) que par des structures créées spécifiquement à cette fin.

L'introduction de ces mesures serait sans doute de nature à modifier certains schémas comportementaux faisant du recours à la violence un mode de résolution de conflit, là où il n'est souvent que le révélateur d'un mode de communication inadapté.

Roger Cantacuzène Docteur en droit Chargé d'enquête au Centre de Recherches sur les Pouvoirs Locaux dans la Caraïbe

(CRPLC / UMR CNRS 8053)

Université Antilles Guyane 


\section{BIBLIOGRAPHIE}

André, J. (1987). L'inceste focal dans les familles noires antillaises, Paris, Presses universitaires de France.

Berger, P. et T. Luckmann (1966). La construction sociale de la réalité, Paris, Armand Colin.

Bougerol, C. (2002). « Vivre en prison à la Guadeloupe. Réputation et virilité chez de jeunes délinquants », Ethnologie française, vol. 32, n² 4, p. 699-708.

Bonniol, J.-L. (1992), La couleur comme maléfice. Une illustration créole de la généalogie des «Blancs » et des « Noirs », Paris, Albin Michel.

Cantacuzène, R. (2011). « Le déficit d'expression de l'intime dans la socialisation masculine aux Antilles ». Communication présentée au colloque international Perspectives futures en intervention, politiques et recherche sur les hommes et les masculinités, Université Laval, Québec, mars 2011.

Cantacuzène, R. (2012). "Les homosexuels aux Antilles, des citoyens de seconde zone ? ». Communication à la $37^{\mathrm{e}}$ Conférence annuelle de la Caribbean Studies Association (Guadeloupe, 28 mai- $^{\text {er }}$ juin 2012).

Du Tertre, J.-B. (1973[1671]). Histoire générale des Antilles habitées par les Français, Fort-deFrance, Éditions Horizons Caraïbes.

Gauthier, F. (2007), L'aristocratie de l'épiderme. Le combat de la Société des Citoyens de Couleur 1789/1791, Paris, CNRS Éditions.

Giraud, M. (1999). «Une construction sociale de la sexualité. À propos du partenariat hétérosexuel caribéen », Actes de la Recherche en sciences sociales, n 128, p. 46-55

Gracchus, F. (1980). Les lieux de la mère dans les sociétés afroaméricaines, Paris, Éditions Caribéennes, C.A.R.E.

Halfen, S. (2006). Les connaissances, attitudes, croyances et comportements face au VIH/sida aux Antilles et en Guyane en 2004, étude ANRS-EN16-KABP-DFA, Paris, ANRS-ORSIF.

Kabile, J. (2011). « Pourquoi ne partent-elles pas ? Les obstacles à la sortie de la situation de violence conjugale », dans N. Lefaucheur (dir.), Pouvoirs locaux dans la Caraïbe, $\mathrm{n}^{0} 17$, « Genre et violences interpersonnelles à la Martinique », Paris, L'Harmattan, p.161-198.

Lefaucheur, N. (dir.) (2011). Pouvoirs locaux dans la Caraïbe, $\mathrm{n}^{0} 17$, « Genre et violences interpersonnelles à la Martinique », Paris, L'Harmattan

[www.editions-harmattan.fr> et sur <www.openeditions.org]

Lefaucheur, N. et L. Ozier-Lafontaine (2011). " Histoires de couples, histoires de violences », dans N. Lefaucheur (dir.), Pouvoirs locaux dans la Caraïbe, n 17, p.125-160. 
Lefaucheur, N. et S. Mulot (2012). « La construction et les coûts de l'injonction à la virilité en Martinique », dans D. Dulong, C. Guionnet, E. Neveu (dir.), Boys don't cry. Les coûts de la domination masculine, Rennes, Presses Universitaires de Rennes, p. 207-229.

Massé, R. (2008). La détresse créole, Québec, Presses de l’Université Laval.

Mulot, S. (2000). " Je suis la mère, je suis le père ! » : l'énigme matrifocale. Relations familiales et rapports de sexe en Guadeloupe. Thèse pour le doctorat, dirigée par Maurice Godelier, Paris, École des hautes études en sciences sociales.

Mulot, S. (2009). « Redevenir un homme en contexte antillais post-esclavagiste et matrifocal », Autrepart, $\mathrm{n}^{\circ} 49, \mathrm{p} .117-136$.

Petitjean-Roget, J. (1980). La société d'habitation à la Martinique. Un demi-siècle de formation 1635-1685. Thèse Université Paris VII (1978), Lille, Atelier de Reproduction.

Pourette, D. (2002). «La figure du makomè, masque de l'homosexualité dans les mondes guadeloupéens », dans R.-M. Lagrave, A. Gestin, E. Lépinard, G. Provost (dir.), Dissemblances. Jeux et enjeux du genre, Paris, L'Harmattan, p. 51-63.

Welzer-Lang, D. (2002). «Virilité et virilisme dans les quartiers populaires en France », VEI Enjeux, $\mathrm{n}^{\circ} 128, \mathrm{p} .10-32$.

Wilson, P. (1969). « Reputation and Respectability : a Suggestion for Caribbean Ethnology», Man, vol. 4, n 1, p. 70-84.

Wilson, P. (1973). Crab Antics. The Social Anthropology of English-speaking Negro Societies of the Caribbean, New Haven, Yale University Press.

Zobda-Zebina, M. (2011). « La construction des marges de la masculinité aux Antilles ». Communication présentée au colloque international Perspectives futures en intervention, politiques et recherche sur les hommes et les masculinités, Université Laval, Québec, mars 2011. 